\title{
AC 2007-2079: A WIRELESS COMMUNICATIONS AND NETWORKING COURSE DEVELOPED FOR ELECTRICAL ENGINEERING AND COMPUTER ENGINEERING TECHNOLOGY PROGRAMS
}

\section{Xuefu Zhou, University of Cincinnati}

Xuefu Zhou is an Assistant Professor of Electrical and Computer Engineering Technology at the University of Cincinnati. He received both his M.S. and Ph.D. degrees in Electrical Engineering from the University of Cincinnati in 2002 and 2006, respectively. He also holds a M.S. degree (1995) in Mechatronics from Huazhong University of Science and Technology. From 1995 to 2000, he worked as an Engineer, Senior Engineer and Project Manager in the high-tech industry on the design, development of Distributed Control Systems (DCS) and Supervisory Control And Data Acquisition (SCADA) systems. His expertise and current research interests lie on wireless communications, wireless and mobile networks, cross-layer design for wireless networks, signal processing, DCS and SCADA systems. He is a member of IEEE and ASEE.

\section{James Everly, University of Cincinnati}

James O. Everly is an Associate Professor of Electrical and Computer Engineering Technology at the University of Cincinnati. He received a BSEE and MSEE from The Ohio State University in 1969 and 1970, respectively. He is a Senior Member of the Institute of Electrical and Electronics Engineers (IEEE) and a registered professional engineer in the state of Ohio. He is currently Chair of the IEEE Cincinnati Section, and in 1997 he received the IEEE Professional Achievement Award. He has held several research and management positions in industry working for such companies as Battelle's Columbus Laboratories, Rockwell International, and Claspan Corporation. He joined the University of Cincinnati in 1985.

\section{Max Rabiee, University of Cincinnati}

Max Rabiee is Professor of Electrical and Computer Engineering Technology (ECET) at the University of Cincinnati. He earned his Ph.D. in Electrical Engineering from the University of Kentucky in 1987. Dr. Rabiee has been a registered professional engineer since 1988, and a senior member of the Institute of Electrical and Electronic Engineering (IEEE). He is also a member of the American Society of Engineering Education (ASEE), the Eta Kappa Nu Electrical Engineering Honor Society, and the Tau Beta Pi Engineering Honor Society. 


\title{
A Wireless Communication and Networking Course Developed for Electrical Engineering and Computer Engineering Technology Program
}

\begin{abstract}
During the past decade, wireless communication has become a ubiquitous technology. The booming application of wireless communication and its fast developing technology have caused significant social and technological impacts. Despite its rising importance, wireless communication and wireless networks are not commonly studied in an Electrical and Computer Engineering Technology program. As a part of our curriculum continuous improvement plan, faculty members in the Electrical and Computer Engineering Technology department at the University of Cincinnati felt it very important to teach students the current wireless and mobile communication technologies, and to let them gain hands-on experiences with the application of wireless technologies. Thus, we proposed a new course to introduce wireless communication and wireless networks in 2006 . The new course has been strongly supported by local industry as well as the department's industrial advisor committee. The new course has been offered twice in 2006 and received very positive student responses. This paper describes the course information, lecture topics, laboratory exercises, student feedback, and the instructor's reflections.
\end{abstract}

\section{Introduction}

Wireless computing is a rapidly emerging technology which offers network connectivity thereby minimizing the need for a wired connection and thus supports the concept of mobility. Wireless technology has already become the most exciting area in telecommunications and networks. The rapid growth of wireless and mobile telephones, satellite communication, wireless local area networks (WLAN), wireless personal area networks (WPAN) and wireless metropolitan area networks (WMAN) and the applications of wireless internet are generating tremendous changes in the telecommunication industry and results in significant impact to the society. For example, businesses are increasing their reliance on wireless networks and developing the requisite infrastructure to implement and integrate wireless technologies into their existing networks. A new market analysis report in November 2006 from The Insight Research Corporation ${ }^{[1]}$ predicts that service revenues in the global telecommunications industry will reach $\$ 1.3$ trillion by the close of 2007, with continued strong growth in wireless leading the way. According to the new industry market study, wireless service revenues are expected to grow at a compounded rate of nearly 10 percent over the next few years, while wired service revenues grow much more modestly at two percent.

Continual growth in the wireless industry can be attributed to several reasons. First, wireless networks provide an alternative to the high installation and maintenance costs incurred by the traditional infrastructure of wired networks. Second, wireless/mobile networks not only offer mobility but also extend the capability to the traditional networks. For example, WLANs can be set up in some environments such as old buildings and museums where traditional wiring is prohibited or impossible. Also, wireless networks have advantages in some operational environments where the network is only operational for a short time. Having gone far beyond 
just the absence of wires, the emergence of various wireless technologies even brought up new definitions of what a network infrastructure can be and ultimately can turn the dream of getting connected "anywhere and at any time" into reality. With the arrival of $3 \mathrm{G}$ cellular networks, WiMAX and new advances in wireless local area networks, an era of wireless technology is approaching.

Although courses on wireless communication and wireless networks have been well developed in most Electrical Engineering, Computer Engineering, and Computer Science programs, they are not commonly offered in Electrical and Computer Engineering Technology (ECET) programs because of the following reasons. First, most of the topics on wireless communications and wireless networks are related to rapidly changing technologies and require theoretical approaches, i.e., requiring advanced mathematical skills, while the majority of ECET programs are taught at undergraduate levels and focus on the practice or the application of current knowledge. Also, instructors often feel challenged to tailor topics to engineering technology programs. Second, the breadth of necessary multidisciplinary understanding often presents significant challenge to students. Third, limited educational resources such as "equipment funding" severally limit the scope of laboratory activities.

Despite these difficulties, our observation of the importance of wireless technologies and the request from the local industry convinced the ECET faculty that it is imperative to incorporate wireless communications and wireless networks into the standard ECET curriculum. This will help our graduates apply state of the art techniques and designs to meet the current needs of society and fill the local wireless industry need.

The course developed in the Winter Quarter of 2006 is named "Introduction to Wireless Communications and Networks." It is offered as a senior required course in the Bachelor of Science in Computer Engineering Technology program and a technical elective course in the Bachelor of Science in Electrical Engineering Technology program. This course consists of three credit hours of lecture and one credit hour of laboratory.

The remainder of this paper is organized as follows: Section 2 discusses course development and describes the context, course objectives, references, and laboratory exercises. Section 3 presents our teaching experiences and reflections and, finally, Section 4 presents conclusions.

\section{Course Development}

\section{A. Context and Objectives}

With the exception of this new course, there are a few existing courses in the ECET curriculum pertinent to communication techniques and networking. They include Electronic Communications, Digital Communications, and RF Communications together with Computer Networks and a three course sequence in Telecommunications.

The first introduction to communications concepts occurs at the sophomore level in Electronic Communications. This course focuses on the introduction of thermal noise concepts together with traditional amplitude and frequency modulation communication techniques. The highlight 
of the course is a construction of a direct conversion short-wave receiver. A direct conversion receiver is used to illustrate the reception of continuous wave (CW) and single-sideband (SSB) signals in the 40-41 meter (7.0-7.3 MHz) short-wave bands. It is the author's opinion that the time and effort spent experimenting with a properly designed double-sideband direct conversion receiver is an integral part of $\mathrm{RF}$ receiver design education. Moreover, a simple direct conversion receiver serves as an important benchmark for comparison and it is useful for student designers to periodically design, and re-design this receiver concept based on advances in technology. Students soon discover that a simple direct conversion receiver is best suited for applications where relaxed selectivity requirements or better sounding audio are the design objectives. The receiver project is implemented with a low-cost, readily available, printed circuit board and two commonly available integrated circuit chips. Electronic assembly time is approximately six hours with a total project cost, excluding resistors and capacitors, under $\$ 25$.

A junior level course on Digital Communications teaches students various digital modulation and detection methods and performance limitations, primarily for data transmission including OnOff-Keying (OOK), Frequency-Shift-Keying (FSK), Quadrature-Phase-Shift-Keying (QPSK), 8 and 16 Phase-Shift-Keying (PSK), and 8 and 16 Quadrature-Amplitude-Modulation (QAM). In this course a class project is used to illustrate concepts introduced in the lecture. A project that has been very successful over the past three years is the integration of a $433.92 \mathrm{MHz}$ wireless temperature sensor into a PSK31 (31 baud) modulated audio beacon transmitter. An audio beacon was chosen so that initially the concept could be tested using an audio rather than a radio frequency (RF) channel. The temperature sensor is a Dallas Semiconductor DS18S20 integrated into standard "off-the-shelf" 433.92 MHz RF transmitter module that uses amplitude modulation. The transmission method known as "On-Off-Keying (OOK)" transmits the temperature data by simply switching the carrier signal on and off. The RF data link serial receiver can be located up to 500 feet from the transmitter and interfaces to the PSK31 Audio Beacon via an RS-232 interface.

The Audio Beacon features PSK31 encoding and audio waveform generation using a single-chip SX-28 RISC microcontroller operating at $50 \mathrm{MHz}$. The beacon has a choice of three base carrier audio frequencies, namely $500 \mathrm{~Hz}, 1 \mathrm{kHz}$, and $2 \mathrm{kHz}$. The audio output is initially fed to an 8 ohm speaker and the encoded audio signal is detected by a microphone attached to a laptop computer running an audio spectrum analyzer software package named Digipan 2.0, available at no cost on the Internet. A recent extension to the project consists of turning the audio single into an RF double-sideband signal using a balanced modulator with carrier frequency of $3.579 \mathrm{MHz}$, and requires a Federal Communications Commission (FCC) license to operate the transmitter. The signal is subsequently reduced to a single-sideband signal by a 3 pole crystal filter. Finally, the signal is further amplified to a power level of 5 watts by a push-pull class AB amplifier. The push-pull amplifier was chosen in order to eliminate second order harmonics and reduce the requirement on the harmonic suppression filter while still complying with FCC specifications. The RF beacon output power is then sequentially reduced by switching in-line a series of $3 \mathrm{~dB}$ attenuators until the output power reaches 0.5 watts. Each time the output power is reduced by 3 $\mathrm{dB}$ the local temperature is updated. The transmit data string consists of station identification, grid square location, return email address, local temperature in ${ }^{\circ} \mathrm{F}$, and output power level. A collection of signal reports from amateur radio operators is used to validate the use of the modulation technique as a low power beacon transmitter. 
An RF Communication course is introduced at the senior level and is based on project-based learning. The course provides a stimulating introduction to analog RF electronics by analyzing the design and construction of a radio transceiver. The course covers key elements of radio electronics, including filters, amplifiers, oscillators, and mixers. The course is designed to lead the student through the design, construction, and testing of a popular high frequency $(7 \mathrm{MHz})$ radio transceiver (the NorCal40A) thereby illustrating and reinforcing the theoretical material presented in the lecture. The course consists of 36 "Problem Exercises" that start with series and parallel resonant circuit concepts and sequentially lead the student through transceiver construction and testing to include a final product demonstration. A low cost vector network analyzer is used to "speed-up" the process of circuit testing. The level of mathematics consists primarily of using "Phasor Concepts" introduced in circuit analysis classes.

Computer Networks gives an extensive introduction to computer networking concepts. It answers the basic question "how do computer networks and internets operate?" in the broadest sense and focuses on concepts, principles and covers all aspects of networking, from low-level details of wiring and signals to applications.

Telecommunications I, II and III focus on the basic concepts of telecommunications systems, emphasizing the present telephone system as well as the new and emerging telecommunications techniques, including cable and satellite systems.

The remainder of this section will focus on the addition of a new course to the curriculum, namely Wireless Communications and Networks. This new course focuses on the wireless/mobile communications and various wireless network technologies. It presents the wireless/mobile transmission fundamentals, wireless and mobile network architectures, technologies and protocols.

Six main objectives of this new course are to:

- Learn the fundamentals of wireless/mobile transmission, wireless/mobile communication technologies;

- Understand the characteristics of wireless links;

- Understand the challenges in designing wireless/mobile networks;

- Become familiar with various wireless and mobile network architectures, technologies and protocols, including 1G, 2G and 3G cellular networks, wireless networks (802.11, 802.15, WiMax);

- Gain hands-on experiences on wireless applications;

- Be able to apply concepts and techniques to senior design projects or other related projects.

To accomplish these objectives, the mathematical contents of the course are treated as a tool to illustrate physical situations instead of as the central core of the course. Topics requiring analytical approaches, such as Fourier series, characterization of channel fading, equalizations and inter-symbol interference etc., are demonstrated through simulations. Conceptual oversimplifications are employed to help students understand the key ideas of the technology. 
While a primary objective of this course is to provide a broad understanding of wireless communication and various wireless networks, several topics such as error detection, error correction coding, are explored in sufficient depth to allow students to develop a solid understanding and be able to apply them to senior design projects involving wireless transmission.

\section{B. References, Course Content}

Although an ideal textbook on Wireless Communication and Networks for Engineering Technology programs is still lacking, W. Stalling's ${ }^{[2]}$ textbook was adopted for this new course. However, a majority of lectures are developed by referring to other texts ${ }^{[3]-[8]}$ as well. There are eight lecture modules in this new course, for about 30 hours of instruction.

The first lecture module (1 hour) starts by reviewing the impact of wireless technology on the society and human life, the advantages of wireless technology, the history of wireless communications and an overview of the challenges of wireless technology such as multipath, fading, limited resources and security issues. It is assumed that most of the students in an engineering technology program have some application experiences (i.e., cell-phones, wireless routers). Thus, one of the objectives of this first lecture module is to help students learn from their own experiences, to reflect on their concrete experiences, and to motivate them to study wireless technology.

The second module ( 3 hours) teaches students the fundamentals of signals and transmission. Specifically, topics include electromagnetic spectrum, frequency, period and phase, wavelength of sinusoid waveforms, analog and digital signaling, Fourier series, channel capacity and the impact of signal-to-noise ratio (SNR) on the wireless communication systems. Basic multiplexing techniques such as frequency division multiple access (FDMA), time division multiple access (TDMA), code division multiple access (CDMA) and space division multiple access (SDMA) are explained. The principle of CDMA is demonstrated by numerical examples.

The third module ( 8 hours) introduces wireless and mobile radio propagation. Topics include antennas, Doppler shift, propagation mechanism (scattering, reflection and diffraction), multipath, channel fading, path loss model, inter-symbol interference (ISI), equalization and basics of detection. Digital modulation schemes such as amplitude-shift-keying (ASK), PSK and FSK, QAM are introduced. After the completion of this lecture module, students should be able to understand the characteristics of wireless links, and the challenges in the design and application of wireless networks.

The forth module ( 3 hours) presents advanced techniques to combat unreliable wireless channels. Topics include error detection, error correction codes (linear block codes, interleaver) and error control mechanisms, i.e., automatic repeat request (ARQ) such as Stop-and-Wait, Go-back-N, and Selective Repeat. Frequency diversity techniques (i.e. spread spectrum, OFDM) and spatial diversity techniques (i.e., multiple antennas) are briefly addressed. Emphasis is placed on why diversity is an effective technique to improve the performance of wireless systems. 
The fifth module (3 hours) introduces cellular networks. Topics include the principle of cellular networks, characterization of frequency reuse, techniques to increase cellular capacity (cell splitting, sectorization, micro cells), soft and hard handoff's, roaming, architecture of cellular networks, the introduction of $1 \mathrm{G}$ (AMPS), $2 \mathrm{G}$ (GSM) and $3 \mathrm{G}$ systems. Also, emphasis is placed on understanding the evolution of cellular networks and the key feature of each cellular network.

The sixth module (7 hours) introduces wireless personal area networks (Bluetooth), wireless local area networks (IEEE 802.11) and WiMAX. Topics on Bluetooth include its operation, application, packet formats, PHY protocol, features and products. Topics on IEEE 802.11 include its features, its physical layers and medium access control layer protocols, application of IEEE 802.11. Topics on WiMAX focus on its future application. Special topics on wireless ad hoc networks and sensor networks are also briefly addressed in this module.

The seventh module ( 3 hours) discusses miscellaneous topics such as radio frequency identification (RFID tags, RFID readers, RFID standards), and GPS and Satellite communications. Emphasis is placed on "what are these technologies", and possible scenarios to apply these technologies.

The eighth module ( 2 hours) introduces wireless security. Various attacks and a case study of wired equivalent privacy (WEP).

\section{Laboratory Exercises}

The initial difficulty with wireless equipment is overcome by running most laboratory exercises by simulations. A brief description of eight laboratory exercises follows. The software program MATLAB is required for most of the exercises.

- Laboratory 1 is developed to have students be familiar with MATLAB program and use MATLAB to generate plots. In addition, students are required to verify Fourier theorem discussed in the lecture, to generate plots to visualize how bandwidth, SNR etc. affect channel capacity.

- Laboratory 2 is developed to have students to model a simple multipath problem by finding signal strength at different location which is resulted from a light of sight (LOS) and a Non-Line-of-Sight (NLOS, reflections from another object such as a large building). This lab illustrates how signal strength varies with locations.

- Students simulate Jake's fading model in Laboratory 3. It illustrates the channel fading and demonstrates how signal strength varies with time.

- In Laboratory 4, students simulate ISI, equalization and understand the impact of wireless channel, noise the performance of detection.

- In Laboratory 5, students simulate a CDMA system. Factors affecting the CDMA performance (path loss, noise, correlations of code sequences) and near-far problem are explored through simulations.

- In Laboratory 6, students are required to implement error detection schemes and error correction schemes and demonstrate how to achieve reliable transmission over unreliable wireless links. 
- In Laboratory 7, students learn how to set up a wireless local area network and how to build a WiFi antenna.

- In Laboratory 8, students use Ethereal to gain hands-on experience with wireless network security.

\section{Student Feedback and Teaching Reflection}

Various methods were used to formally assess the effectiveness of this new course, including tests, the evaluation of student work, and the instructor's assessment of laboratory work. Based on students' feedback, the overall response from students regarding whether the course met their expectations was very positive. Summarized student comments are:

- This course presents interesting topics and helps them to learn new technologies.

- They have a better understanding of wireless and mobile devices

- The course covers wide topics related to wireless communication and networking.

- They feel confident to integrate wireless technology in their senior design projects.

- They are more interested in looking for a job in the wireless industry.

Also received are comments on the improvement of the course, mainly on how to incorporate more practical application projects into the laboratory. In addition, some students felt that this course was too demanding---too many topics packed in this course. Thus, consideration is being given to dividing the material into two courses. One of the courses will focus on Wireless Communications and the other will focus on Wireless Networks.

\section{Conclusion}

Recently, wireless technology market has experienced unprecedented growth. It is critical to include the wireless technology into our Electrical and Computer Engineering Technology curriculum. This paper provides a brief summary on a newly developed course on wireless communication and networking. Overall, response from students shows that this new course is successful in helping them learn new technology and practice in a new field.

\section{References}

1. Online research, http://www.insight-corp.com/

2. W. Stalling, Communications and Networks, 2nd Ed., Prentice Hall.

3. IEEE 802.11 standards

4. IEEE 802.15 Standard

5. IEEE 802.16 Standard

6. T. Rappaport, Wireless Communications: Principles and Practice, Prentice Hall, 1999, 2002

7. V. Garg, Wireless Network Evolution:2G to 3G, Prentice Hall, 2002

8. J. Landt, Shrouds of Time: outlines history and present of RFID: http://www.aimglobal.org/technologies/rfid/resources/shrouds_of_time.pdf 